\title{
Doenças crônicas e o acometimento por COVID-19: cuidados de enfermagem
}

\author{
Chronic diseases and covid-19 affairment: nursing care \\ Enfermedades crónicas y afecciones por covid-19: cuidado de enfermería
}

\section{RESUMO}

Objetivo: analisar publicações científicas referentes aos cuidados de enfermagem para as pessoas com doenças crônicas e o acometimento por Covid-19. Método: Revisão integrativa da literatura, descritiva, exploratória, de origem retrospectiva. Realizado nas bases de dados da SciELO-Scientific Electronic Library Online e Google acadêmico. Utilizando os descritores: Doença crônica; Covid-19; Enfermagem; Cuidado e Pandemia, cruzados pelo operador booleano AND, conforme os critérios de inclusão com estudos entre os anos de 2019 e 2021 . Foram eleitas 10 publicações referentes ao tema e organizadas em um quadro para melhor compreensão do assunto em questão. Resultados: As publicações inseridas na pesquisa foram realizadas todas no ano de 2020, por garantirem confiabilidade aos estudos e consequentemente credibilizarem o desempenho da enfermagem. Conclusão: Os cuidados de enfermagem são de suma importância no tratamento de pacientes com doenças crônicas e com acometimento por Covid-19.

DESCRITORES: Doença crônica; Covid-19; Enfermagem; Cuidado; Pandemia

\section{ABSTRACT}

Objective: to analyze scientific publications related to nursing care for people with chronic diseases and involvement by Covid-19. Method: Integrative literature review, descriptive, exploratory, retrospective in origin. Carried out in the databases of SciELO-Scientific Electronic Library Online and Academic Google. Using the descriptors: Chronic disease; Covid-19; Nursing; Care and Pandemic, crossed by the Boolean operator AND, according to the inclusion criteria with studies between the years 2019 and 2021.10 publications related to the topic were chosen and organized in a framework for a better understanding of the subject in question. Results: The publications included in the research were all carried out in 2020 , as they ensured reliability to the studies and consequently gave credibility to the nursing performance. Conclusion: Nursing care is of paramount importance in the treatment of patients with chronic diseases and affected by Covid-19.

DESCRIPTORS: Chronic disease; Covid-19; Nursing; Care; Pandemic.

\section{RESUMEN}

Objetivo: analizar las publicaciones científicas relacionadas con la atención de enfermería a las personas con enfermedades crónicas y la implicación del Covid-19. Método: Revisión bibliográfica integradora, descriptiva, exploratoria, de origen retrospectivo. Realizado en las bases de datos de SciELO-Scientific Electronic Library Online y Academic Google. Utilizando los descriptores: Enfermedad crónica; COVID-19; Enfermería; Care and Pandemic, atravesado por el operador booleano AND, de acuerdo con los criterios de inclusión con estudios entre los años 2019 y 2021. Se eligieron y organizaron 10 publicaciones relacionadas con el tema en un marco para una mejor comprensión del tema en cuestión. Resultados: Las publicaciones incluidas en la investigación fueron todas realizadas en 2020, ya que aseguraron confiabilidad a los estudios y consecuentemente dieron credibilidad al desempeño de enfermería. Conclusión: El cuidado de enfermería es de suma importancia en el tratamiento de pacientes con enfermedades crónicas y afectados por Covid-19.

DESCRIPTORES: Enfermedad crónica; COVID-19; Enfermería; Atención; Pandemia.

RECEBIDO EM: 08/06/21 APROVADO EM: 14/06/21

\section{Kátia Cristina Barbosa Ferreira}

Enfermeira. Universidade Estadual da Paraíba.

ORCID: 0000-0002-3353-2973.

\section{Luana Gomes Leitão Rodrigues}

Enfermeira. Centro Universitário de João Pessoa.

ORCID: 0000-0001-5259-1357. 
Luciene de Souza Santos Albuquerque

Enfermeira. Centro Universitário de João Pessoa.

ORCID: 0000-0001-5119-195X

\section{Maria Clara Ribeiro Costa}

Enfermeira. Centro Universitário de João Pessoa.

ORCID: 0000-0003-4422-3719, Paraíba.

\section{Wanessa de Araújo Evangelista}

Pós-graduanda em Emergência e UTI (FESVIP), Pós-graduanda em Instrumentação Cirúrgica, Centro Cirúrgico e Central de Material e Esterilização (FAVENI).

ORCID: 0000-0002-5702-5634.

\section{Aline Pereira da Silva}

Enfermeira. Pós-granduanda em obstetrícia e neonatologia. Faculdade de Enfermagem Nova Esperança. ORCID: 0000-0001-8185-9471.

\section{INTRODUÇÃO}

A Organização Mundial da Saúde (OMS) define as patologias crônicas como doenças de lenta progressão e longa duração. Podem ser silenciosas ou sintomáticas, comprometendo a qualidade de vida do paciente por toda a sua vida $(1,2)$. As doenças crônicas são patologias de múltiplas causas e fatores que constituem um grave problema de saúde pública. No Brasil, representam a maior causa de mortes na população(2).

Os dados da Pesquisa Nacional de Saúde (PNS) apontam que cerca de $40 \%$ dos brasileiros, cerca de 57 milhões de pessoas, sofriam de pelo menos uma doença crônica, em 2013(1). A Covid 19 é uma infecção respiratória aguda causada pelo SARS- $\mathrm{CoV}-2$, de elevada transmissibilidade e de distribuição global. O SARS-CoV-2 é um betacoronavírus que pertence ao subgênero Sarbecovírus da família Coronaviridae e é o sétimo coronavírus conhecido a infectar seres humanos(3).

As doenças crônicas são as principais comorbidades dos pacientes com COVID-19, sendo responsáveis pelas complicações e agravamento da condição clínica do paciente, gerando uma elevação do tempo de internação e das taxas de mortalida$\operatorname{de}(4,5)$.

A enfermagem tem responsabilidade com ações e práticas de educação, promoção à saúde, prevenção das doenças crônicas e orientações diante dessa pandemia, com o intuito de buscar uma melhor qualidade de vida para os seus pacientes. Essas características são essenciais para o cuidado à saúde das pessoas com doença crônica, pois as ações de promoção à saúde fornecem um olhar sobre a doença, evitam complicações e melhoram a qualidade de vida das pessoas, assim como das famílias $(5,6,7,8)$.

Sendo assim, levantou-se a seguinte questão de pesquisa: $\mathrm{O}$ que se tem na literatura atual, a respeito dos cuidados de enfermagem em relação as pessoas com doenças crônicas acometidas pelo Covid-19? Desse modo, tem-se por objetivo: analisar publicaçôes científicas referentes aos cuidados de enfermagem para as pessoas com doenças crônicas e o acometimento por Covid-19.

\section{MÉTODO}

Esta pesquisa é de cunho descritivo, exploratória, de origem retrospectiva, na modalidade de revisão integrativa. $\mathrm{O}$ delineamento do estudo foi construído seguindo seis etapas: período de coleta de dados, limite temporal estabelecido para a pesquisa, bases elegíveis para a busca de publicações, critérios de inclusão/exclusão, uso de operadores booleanos, uso de um instrumento autoral, análise de dados e validação da busca. Realizada no mês de maio, ano de 2021, nas bases de dados da SciELO-Scientific Electronic Library Online e Google acadêmico. Foram adotados como critérios de inclusão: artigos no idioma português brasileiro, disponíveis integralmente, de forma gratuita, sem duplicidade no momento da busca na base de dados, e que estejam dentro do período estabelecido pela pesquisa, sendo de 2019 a 2021. Foram excluídos da pesquisa: os estudos que não corresponderam aos objetivos da pesquisa e não estavam nos critérios de inclusão.

Para a seleção das publicações, foi utilizado o operador booleano AND, com os descritores em português: Doença crônica; Covid-19; Enfermagem; Cuidado e Pandemia. Estes descritores foram cruzados de diferentes formas, buscando abranger mais o tema. Os dados foram analisados utilizando o programa da Microsoft Word, e as publicações foram expostas em um quadro, contendo: autores, o título, ano de publicação, periódicos e aspectos relevantes aos cuidados de enfermagem para às pessoas com doenças crônicas e acometidas pela covid-19, descritas nas publicações. A pesquisa não foi submetida ao Comitê de ética em pesquisa, por se tratar de uma pesquisa na literatura.

No total, foram encontradas 195 publicações científicas sobre a temática. Após a realização da filtragem por ano de publicação (2019 a 2021), permaneceram 195 artigos, pelo motivo que a pandemia é algo recente no mundo, tornando as publicações atuais. Em seguida, foram lidos os títulos das publicaçôes, no qual foram excluídas 155 publicações, por não responderem aos 


\section{artigo}

Ferreira, K. C. B., Rodrigues, L. G. L., Albuquerque, L. S. S., Costa, M. C. R., Evangelista, W. A., Silva, A. P.

Doenças crônicas e o acometimento por covid-19: cuidados de enfermagem

objetivos do estudo.

Dando sequência a pesquisa com 40 publicações, foram lidos os resumos, e analisados minuciosamente por todos os autores. A partir disso, percebeu-se que apenas 10 publicações tratavam do que as autoras gostariam de abordam sobre um tema tão amplo. Desse modo, as 10 publicações foram lidas integralmente, para confirmar os achados científicos e validar a seleção das publicações. Permaneceram na análise ao final, 10 publicações científicas. As publicações foram fichadas usando um instrumento de autoria dos pesquisadores deste artigo, que continha: autores, o título, ano de publicação, periódicos e aspectos relevantes aos cuidados de enfermagem para às pessoas com doenças crônicas e acometidas pela covid-19. A busca foi validada por dois pesquisadores. Os dados da pesquisa foram organizados em um quadro, figura essa que, detalha os principais achados das $10 \mathrm{pu}-$ blicações científicas selecionadas, trazendo uma visão organizada, ampla e compreensível do assunto em questão.

\section{RESULTADOS}

Após a análise, foi construído o quadro abaixo, visando melhor exposição dos resultados encontrados.

As pesquisas foram realizadas no ano de 2020, e publicadas em periódicos que garantem confiabilidade aos estudos, e con- sequentemente, credibilizam o desempenho da enfermagem. Apresentam dados do desenvolvimento clínico de pacientes brasileiros, com base em experiências de outros países, de forma abrangente, com relação às categorias de profissionais envolvidos e direcionadas, em sua maioria, aos pacientes com comorbidades.

Apesar das poucas citações de intervenções específicas de enfermagem, é possível identificar a importância da classe no enfrentamento da pandemia em todos os níveis de assistência. Ações bem presentes na educação em saúde, passando pelo monitoramento, controle, assistência direta ao paciente e gestão de fluxo em unidades de saúde.

\section{DISCUSSÃO}

$\mathrm{Na}$ análise dos artigos selecionados, é possível perceber que a ação de enfermagem é de suma importância para o enfrentamento da Covid-19. O olhar atento e a tomada de decisão voltada ao público mais frágil, indivíduos esses, que já são comprometidos por possuírem comorbidades, é fundamental que prestação de serviço de saúde tenha como foco sempre a preservação da vida(6).

$\mathrm{O}$ coronavírus trouxe um impacto muito grande para a população portadora de doenças crônicas, esse público necessita de uma atenção diferenciada. O resguardo dos indivíduos portadores de patologias, tanto de forma individual quanto coletiva, diminui as chances da doença em questão alcançar essas pessoas, uma vez que, a sociedade adotando medidas preventivas e agindo com responsabilidade, pode reduzir os índices de morbimortalidade(6). A capacitação dos profissionais de saúde, especialmente os profissionais de enfermagem, fortalece as ações de educação em saúde. A gestão pública deve estar engajada na tomada de decisões e promoção de ações que organize de forma eficaz e priorize a população de risco(6).

A Covid-19 é uma doença que alcança qualquer público, seja ele, criança, jovens, adultos ou idosos, todos estão passíveis de serem acometidos por essa doença. Todavia, o idoso que possua doença cardiovascular infectado por Covid-19, pode manifestar um quadro ruim no tocante ao prognóstico. A prevenção é a forma mais recomendada e pode diminuir a propagação do vírus, como: evitar lugares aglomerados, lavar sempre as mãos e usar álcool em gel à 70\%, são algumas das medidas importantes para se obter um melhor controle da doença(7).

A população de baixa renda está mais vulnerável à crise de saúde pública, por ter a chance mais acentuada de se precisar de internação quando contaminada pelo Covid-19. Cerca de $80 \%$ das mortes de forma geral, de pessoas com doenças crônicas, sucede em países de baixa renda. Para se evitar o colapso no sistema de saúde e a aceleração

QUADRO 1- Apresentação das publicações científicas referentes aos cuidados de enfermagem para às pessoas com doenças crônicas acometidas por COVID-19:

Autores

Título

COVID-19 e doenças

Estrela,FM. et al (6).

Ferrari, F. (7)

Carvalho L, Xavier

LL, Pires LN (8)

crônicas: impactos e pandemia.
COVID-19 e desigualdade no Brasil. desdobramentos frente à

COVID-19: dados atualizadose sua relação com o sistema cardiovascular.

2020

Ano de publicação

Periódico

2020

Revista Baiana de Enfermagem.

Arquivos Brasileiro de Cardiologia.
Cuidados de enfermagem para às pessoas com doenças crônicas e a COVID-19.
2020

Experiment Findings.
- Monitoramento de SSVV em paciente de UTI. - Monitoramento ácido-base.- Administração de medicamentos.- Oferta de $\mathrm{O} 2$ 
Strabelli TMV, Uip

DE.(9)

COVID-19 e o coração.

2020

Arquivos Brasileiros de Cardiologia.

COVID-19 e doença hipertensiva no Brasil: possibilidades de uma tempestade perfeita.

2020 TB, Grezzana GB; Stein AT.(10)

Dantas TP et al. (12)

Diagnósticos de enfermagem para pacientes com COVID-19.

Educação como intervenRodrigues RCR et al.(13) ção de enfermagem na prevenção de COVID
em diabéticos.

Tecnologia móvel para

Neves DM et al.(14)

Pereira FAC, Correia DMS.(15) cuidado de enfermagem durante a pandemia da COVID-19.

Uso da teleconsulta pelo enfermeiro a cardiopatas: uma reflexão durante pandemia por COVID-19 no Brasil.
Sociedade Brasileira de Epidemiologia.
Departamento de Ciências e Tecnologia da Universidade Aberta.

2020 Journal Health NPEPS.

2020

Revista Brasileira de Educação e Saúde.

2020

Enfermagem em Foco.

2020 Enfermagem em Foco.
- Monitoramento de SSVV em pacientes de UTI.- Realização de exames.- Monitoramento ácido-base.

- Controle de doenças crônicas na APS.

- Educação em saúde (foco em estilo de vida saúdável).- Consultas de enfermagem através da telemedicina.

- Oferta de 02.- Reposição eletrolítica.Mudança de decúbito.- Monitoramento de SSVV.- Administração de medicamentos.

- Educação em saúde.- Controle de doenças crônicas.

- Monitoramento de pacientes com doenças crônicas através da telemedicina.- Educação em saúde.- Reorganização do fluxo da unidade de saúde.

Encaminhamento através da teleconsulta.- Orientações através da teleconsulta.

Fonte: dados da pesquisa, 2021.

do índice de óbitos, deve-se adotar medidas que não só combata a contaminação pelo vírus, mas que facilite a vida dos indivíduos pobres, para que eles consigam se prevenir e ao mesmo tempo ter uma renda que os sustentem(8).

Estudos vem mostrando uma grande relação entre a Covid-19 e as complicações cardíacas(16). Um estudo apontou que de 138 pacientes internados por esse vírus, 16,7\% evoluíram para arritmia e 7,2\% manifestaram lesão cardíaca aguda. O Council on Hypertension of the European Society of Cardiology, recomenda que médicos façam o tratamento com anti-hipertensivo usual para pacientes com covid-19(9).

É de suma importância, que a atenção à saúde, trabalhe precocemente, para suprir as necessidades de indivíduos hipertensos, principalmente se tratando da atenção primária. Sabendo que o distanciamento social é uma medida para minimizar a transmissão da Covid-19, a busca por atendimento à saúde fica menos acessível à essa popula- ção em questão, usar meios de atendimento como a telemedicina, bem como facilitar a entrega de medicamentos, são medidas que podem diminuir o risco de contaminação. São formas de fortalecer a estratégia de saúde e continuar promovendo assistência precoce e de qualidade(10).

Para o bem-estar físico e psicológico, é fundamental investir no estilo de vida saudável. Além das medidas preventivas para o enfrentamento da transmissão do Covid-19, como, o distanciamento social, uso 


\section{artigo}

de máscaras de proteção e uma boa higienização pessoal, pode-se dizer que, o cuidado principalmente de indivíduos com doenças crônicas ou com pré-disposição, tanto para prevenir quanto para promover uma melhoria no estado de saúde, se faz necessário adquirir medidas como uma boa nutrição, atividades físicas e uma boa qualidade de sono, medidas estas, que beneficia de forma positiva a resposta imunidade do indivíduo(11). Segundo Dantas et al (12), os principais sintomas da Covid-19, são: tosse, mialgia, hipertermia e expectoração ativa. Os autores observaram, que de 25 diagnósticos de enfermagem, prevaleceram os diagnósticos: proteção ineficaz relacionada à incapacidade de proteção contra agente infeccioso evidenciado por tosse, calafrios e fadiga, dor muscular, dor muscoequelética; padrão respiratório ineficaz relacionado a dor e fadiga evidenciado por dispneia e sofrimento espiritual relacionado a depressão, estressores e mudança ambiental evidenciado por insônia. A educação em saúde é um ponto muito importante, principalmente se tratando de pessoas portadoras de doenças crônicas como por exemplo a Diabetes Mellitus. Implementar medidas de orientações, utilizando-se de tecnologias em saúde, colabora para o aperfeiçoamen- to da promoção em saúde. A enfermagem está diretamente compromissada com a prevenção de doenças, como a Covid-19, promovendo assim, estratégias educativas de autocuidado(13).

Atualmente, a Covid-19 está em processo de espalhamento extremamente acentuado, faz-se necessário cada vez mais a utilização de meios tecnológicos remotos para garantir a continuidade da assistência em saúde. O uso de tecnologia móvel, torna possível o atendimento da enfermagem eficaz, possibilita o acesso dos profissionais de saúde aos pacientes mais vulneráveis(14).

É possível fazer orientações através da tecnologia de teleconsultas a pacientes de alto risco, sabendo que indivíduos com doenças crônicas como os cardiopatas são pessoas vulneráveis e que necessitam de uma maior atenção, o atendimento garante que o profissional passe informações no tocante a possíveis tratamentos, orienta a importância de se manter o isolamento social, bem como fornece medidas com a finalidade de prevenir a contaminação pela Covid-19(15).

\section{CONCLUSÃO}

Esta pesquisa atingiu o seu objetivo, catalogando os cuidados de enfermagem em doenças crônicas e em acometimentos covid-19 de forma organizada, perante publicações atuais inerentes ao tema, fortalecendo a prática de enfermagem e saúde. Os principais cuidados de enfermagem encontrados foram: consulta de enfermagem e monitoramento por telemedicina, monitoramento de SSVV em paciente de UTI, monitoramento de ácido-base, administração de medicamento, oferta de $\mathrm{O} 2$, controle de doenças crônicas, realização de exames, mudança de decúbito, reposição eletrolítica e educação em saúde. Todos os estudos analisados destacaram a necessidade e a importância do enfermeiro no cuidado continuado de paciente com doenças crônicas e covid-19.

A enfermagem tem um importante papel no combate ao covid-19, os enfermeiros se destacam como atores principais dessa trágica pandemia. Esse protagonismo se deve pela atuação corajosa, efetiva e interrupta dentro e fora dos hospitais, prestando uma assistência integral e qualificada. Resultando na possível recuperação e reabilitação dos pacientes, através de cuidados específicos e da sistematização de enfermagem.

\section{REFERÊNCIAS}

1- Sociedade Mineira De Reumatologia. 2019.

2- Figueiredo BEA, Ceccon FR, Figueiredo CHJ. Doenças crônicas não transmissiveis e suas implicações na vida de idosos dependentes. Ciência \& Saúde Coletiva. v. 26, n. 1, p. 77-88, 2021.

3- Brasil. Ministério da Saúde. Governo Federal, 2021.

4- Malta DC. et al. Doenças crônicas não transmissiveis e mudanças nos estilos de vida durante a pandemia de COVID-19 no Brasil. Revista Brasileira de Epidemiologia. v. 24, p. 1-15, 2021.

5- Becker RM, Heidemann ITSB, Meirelles BHS, Costa MFBNA, Antonini FO, Durand MK. Nursing care practices for people with Chronic Noncommunicable Diseases. Rev Bras Enferm. 2018;71(Suppl 6):2643-9.

6- Estrela,FM. et al. COVID-19 e doenças crônicas: impactos e desdobramentos frente à pandemia. Revista Baiana de Enfermagem. 2020.

7- Ferrari, F. COVID-19: dados atualizadose sua relação com o sistema cardiovascular. Arquivos Brasileiro de Cardiologia. 2020.

8- $\quad$ Carvalho L, Xavier LL, Pires LN. COVID-19 e desigualdade no Brasil. Experiment Findings. 2020.

9- Strabelli TMV, Uip DE. COVID-19 e o coração. Arquivos Brasileiros de Cardiologia. 2020.
10- Melo DO, Ribeiro TB, Grezzana, GB; Stein AT. COVID-19 e doença hipertensiva no Brasil: possibilidades de uma tempestade perfeita. Sociedade Brasileira de Epidemiologia. 2020.

11- Vaz PF. COVID-19 e estilo de vida saudável. Departamento de Ciências e Tecnologia da Universidade Aberta. 2020.

12- Dantas TP et al. Diagnósticos de enfermagem para pacientes com COVID-19. Journal Health NPEPS. 2020.

13- Rodrigues RCR et al. Educação como intervenção de enfermagem na prevenção de COVID-19 em diabéticos. Revista Brasileira de Educação e Saúde. 2020.

14- Neves DM et al. Tecnologia móvel para cuidado de enfermagem durante a pandemia da COVID-19. Enfermagem em Foco. 2020.

15- Pereira FAC, Correia DMS. Uso da teleconsulta pelo enfermeiro a cardiopatas: uma reflexão durante pandemia por COVID-19 no Brasil. Enfermagem em Foco. 2020.

16- BCP Costa, MRS Sudré. Pandemia COVID-19 e sua relação com a doença cardiovascular: revisão integrativa. Revista saúde coletiva de Barueri. 2020. 